\title{
Diagnosing the formation of an environment- oriented component of competencies in higher education
}

\author{
Olga Ponomariova ${ }^{1}$, Olga Vasina $^{2,}{ }^{*}$, Sergey Shurygin ${ }^{3}$ \\ ${ }^{1}$ A.V. Khrulev Penza Branch of the Military Academy of Logistics, Department of Humanities and \\ Socioeconomic Disciplines, 440005, Penza, Russia \\ ${ }^{2}$ Penza State University, Department of General Biology and Biochemistry, 440026, 40 Krasnaya \\ Street, Penza, Russia \\ ${ }^{3}$ A.V. Khrulev Penza Branch of the Military Academy of Logistics, 440005, Penza, Russia
}

\begin{abstract}
The article examines the diagnostic tools for evaluating the formation of an environment-oriented component of competencies in students within the system of higher education. The authors present the set of diagnostic tools following their long-term work on identifying the experience of emotional and values-based attitude to nature as the most difficult element of the environment-oriented component of competencies to assess. The article substantiates the selection of diagnostic tools taking into account available environmental knowledge, skills, abilities and behavioural stereotypes of students. It focuses on determining the level of adapted social experience combining the experience of environmentoriented cognitive activity, environment-oriented work methods, environment-oriented creative activity, emotional and values-based attitude. The use of diagnostic tools is illustrated by experimental work within the educational process at A.V. Khrulev Penza Branch of the Military Academy of Logistics and Penza State University.
\end{abstract}

\section{Introduction}

The requirements for identifying the structural and content aspects and diagnosing the environment-oriented component of competencies provide for the scientific understanding of its formation as a newly formed integrated personality structure [11; 12]. Since 2016, a group of researchers from Penza State University, Omsk Branch of the Military Academy of Logistics and A.V. Khrulev Penza Branch of the Military Academy of Logistics has been conducting research in the field of environmental education in higher education institutions $[12 ; 13 ; 15 ; 19 ; 20]$. The authors have significant experience in similar activities, which was initiated by O.N. Ponomariova in the late 1990s following the diagnosis of secondary school children $[1 ; 16]$. The scope of research is the environment-oriented component of competencies containing the following elements: knowledge, skills and abilities, shaped stereotyped behaviours, capabilities in progress, and crystallised volitional efforts of a

*Corresponding author: onvasina@yandex.ru 
person. The focus of research is the diagnosis of forming the environment-oriented component of competencies, including:

- evaluating results of cognitive activity and experience assimilation to employ the known work methods, which is fixed in the form of learning outcomes, i.e. skills and abilities to act according to the model (academic performance of students);

- measuring learning outcomes by means of monitoring social, moral, and emotional qualities of a person (e.g. decision-making and problem-solving skills);

- examining psychological qualities and newly formed personality (development of values, attitudes, and behaviours).

\section{Theory and research methodology}

The methodological basis of research is competence-based, cultural and systemic approaches. It focuses on identifying, developing and selecting diagnostic tools to evaluate the formation of the environment-oriented component of competencies in the process of higher education. The diagnosis allows recognising and assessing existing environmental knowledge, skills, abilities, capabilities and stereotyped behaviours in students. It characterises the state (result) of the formation of the environment-oriented component of competencies. By providing relevant feedback, the diagnosis has control (obtaining data), prognostic (predicting changes in the development of students), corrective and educational functions. An important element of the diagnosis is monitoring the process of assimilating knowledge, skills, and abilities. The assessments ensures receiving feedback and obtaining information about learning outcomes.

The forms of assessment in terms of qualitative and quantitative analysis include: 1) awarding grades (according to the five-point grading scale, where 5 is excellent, $4-$ good, 3 - satisfactory, 2 - unsatisfactory, and additionally passed and failed) and points (100point-rating system); 2) evaluating psychodiagnostic indicators that have an independent prognostic value (analysing and interpreting the results of monitoring).

The types of assessment in the educational process are diagnostic assessment, formative assessment, summative assessment, and confirmative assessment. In addition, students are certified with regard to their professional level. They pass qualifying examinations and write theses (within their study fields). The projective methods of psychodiagnosis are also used.

The assessment is targeted at motivation and stimulation of students, collection of primary data for their academic performance evaluation, correction of mistakes, allocation of grades, and feedback (including the measurement of learning outcomes) $[13 ; 15 ; 19]$.

The formation of the environment-oriented component of competencies has high social and cultural significance with reference to prioritising the preservation of life, maintaining the stability of the biosphere as a global ecosystem and understanding the strategic social and economic objectives of environmentally sustainable development in Russia until 2030 (S.V. Alekseev, S.N. Glazachev, E.N. Dzyatkovskaya, D.S. Ermakov, A.N. Zakhlebny, A.V. Ivashchenko, I.V. Moiseeva, G.A. Paputkova, I.V. Petrukhina, I.E. Pistunova, I.N. Ponomariova, O.N. Ponomariova, O.G. Rogovaya, G.P. Sikorskaya, N.V. Skalon, E.A. Tomakov and others) $[5 ; 6 ; 8 ; 12 ; 17]$.

Diagnosing the formation of the environment-oriented component of competencies aims at measuring the level of adapted social experience, which harmonises the experience of environment-oriented cognitive activity, environment-oriented work methods, environment-oriented creative activity, emotional and values-based attitude to nature, society, homeland, profession, and health (I.Ya. Lerner, V.V. Kraevsky, V.V. Nikolina, O.N. Ponomariova) [2;12]. Consequently, the set of diagnostic tools is designed to use 
available environmental knowledge, skills, abilities and behavioural stereotypes of students. It includes:

- assessing cognitive activity of a person in terms of his / her learning outcomes, i.e. knowledge about nature, society, technology, human, work methods;

- evaluating experience in applying known work methods in terms of learning outcomes, i.e. skills and abilities to follow the model;

- examining experience of creative activity in terms of learning outcomes, i.e. ability to come up with non-standard solutions in difficult situations;

- diagnosing emotional and values-based attitude in terms of personal development of the paradigm of values, attitudes and behaviours, including the emotional space of a person $[2 ; 7 ; 9 ; 18]$.

Testing (including computer testing) allows diagnosing the formation of environmental knowledge and skills. Test as a system of standardised tasks automates the procedure for assessing knowledge and skills of students. Test results reveal:

- assimilation of environmental terms / concepts included in the test (which are learned better and which are learned worse) and environment-oriented material related to different academic disciplines / modules;

- features of ecological thinking of a person at different stages of formation and development through analysing the features of understanding the semantic content of tasks and correlating the content of tasks with the environment-oriented content of various disciplines / modules and life experience of students.

The quantitative and qualitative analysis of test results indicates typical mistakes, least assimilated content, and level of ecological thinking. It allows outlining the ways of adjustment and improvement within the educational process.

Information about the social, moral, and emotional qualities of a person and his / her ability to make non-standard decisions in problem situations can be collected using some interactive teaching methods. These methods are characterised by integrating procedures for the transfer of (educational) experience and procedures for the determination of learning outcomes: game-based learning (role-playing, adventure games, didactic games, escape rooms, computer games, etc.) and nongame-based learning (discussions, debates, case study, etc.) [2, 3]. It presupposes effective interpersonal communication between teachers and students and among students.

This teaching method is built in the form of a trusting (in relation to all participants) exchange of views. Such free discussion is only possible if the teacher has authority and respect as a personality and a professional. It is the way of delivering classes that the scientific, ethical and values-based ideas and views are adjusted, the erroneous standpoints of students are overcome, the focus on tolerating and respecting other people's opinions is stimulated. Discussions can take place in various modes, including with a high level of problematicity (ping-pong discussion (question-and-answer mode), guided conversation (directed dialogue), collaborative learning methods, etc.).

The use of the discussion method requires high proficiency in the study field and brilliant management of the educational process. For instance, the teacher should have the professional skills similar to those of TV presenters and talk show hosts to arrange a heuristic conversation and create conditions for active discussion during a practical class. Though, the result of a properly held discussion in the classroom is not public (if the class is not recorded), but it is always a building block for the personality development. More often, teachers conduct training in a monologue form, since multi-person dialogue / polylogue requires higher level of thinking and speaking of both the teacher and the student. Teachers are more accustomed to a monologue than to a question-answer polylogue, which is inconvenient and the results of which are difficult to predict. In the discussion mode that has been used since the ancient times as one of the basic forms of 
teaching (the galaxy of the greatest teachers of the past brilliantly mastered it), the role of the teacher can vary from the leading to the supporting one ("behind-the-scenes"). However, the success and satisfaction with the discussion among its participants largely depend on the teacher's background, his / her outlook and intelligence.

Within environmental education, the case study method (a type of problem-based learning) is grounded on handling and examining environment-oriented situations (engineering, teacher training, economic, managerial, ecological, etc.) as a task. Students first analyse the presented case, produce and suggest possible solutions, then choose the most suitable one, although in some cases there may be multiple correct solutions. The task is aimed at finding a solution to various situations (cases) that have happened or may potentially happen:

- choice case (there is a number of ready-made solutions, including the wrong ones, students need to choose the correct one);

- $\quad$ illustration case (the patterns, mechanisms, and effects should be described);

- problem case (a true situation is given, students should find a way out or to conclude that there is no way out);

- refutation case (students need to prove the inconsistency of any idea);

- assessment case (the situation has a favourable outcome, students should critically analyse the found solution);

- $\quad$ exercise case (students gain experience through processing professional data, as well as develop the ability to work with publicistic and scientific literature, reference books) $[2,3]$.

The systemic and systematic use of case tasks in the educational process contributes to the formation, development and improvement of various personal (professional) qualities in students, including: interpersonal communication skills for successful interaction and collective decision-making, presentation skills, professional skills and abilities, information skills, and problem-solving skills $[13 ; 15 ; 19 ; 20]$.

In modern education around the world, the following methods are recognised as reliable and valid for diagnosing the development of emotional and values-based attitudes: diagnosis of ecological awareness by means of subtests to assess unity with nature and to measure environmental responsibility (V.I. Panov); completion of sentences (O.N. Ponomariova); Naturephile, EKPB (emotion, knowledge, protection, benefit), Alternative (S.D. Deryabo, V.A. Yasvin); Dominant (A.F. Lazursky, S.L. Frank, S.D. Deryabo, V.A. Yasvin); DeGroot to establish the egoistic, altruistic and biospheric value orientations in emotional and values-based attitude of a person $[4 ; 10 ; 12 ; 16 ; 20]$.

\section{Results and findings}

The authors developed a didactic set "Ecology" consisting of six items (including Ecology. Study Guide, Ecology. Drill Book and Ecology. Teacher's Book) [3; 14]. These materials provide test tasks for formative and summative assessment as a basis to estimate the acquisition of environmental knowledge and skills. The tasks (namely, case tasks and tasks for roundtable discussions, SWOT analysis, and brainstorming) are structured according to the existing environmental problems and challenges. The indicators of forming the environment-oriented component of competencies (based on the participant observation under distance learning) are as follows:

1. The student is able to identify and evaluate the key elements in the task (Yes / No);

2. The student is able to organise team work on solving a (standard / non-standard environmental) problem (Yes / No);

3. The student is able to think critically and logically when solving problems (Yes / No); 
4. The student is able to reason and justify the findings (Yes / No);

5. The student statements are explicit and meaningful (Yes / No);

6. The student behaviour, remarks and statements show that he / she is able to go beyond the task under discussion (Yes / No);

7. The student behaviour, remarks and statements show that he / she applies available data and resources (Yes / No);

8. The student demonstrates knowledge of the environmental effects of the problem (Yes / No);

9. The student is able to maintain positive psychological environment in the team to solve the problem (Yes / No);

10. The student is able to make the right decision under the discussion / case study (Yes / No) [15].

These elements allow diagnosing experience in applying known work methods and maintaining creative activity. However, the data obtained are not enough to identify the motives (reasons) that determine the choice of a particular behaviour pattern, expressed opinion, value judgment, action, which characterises the peculiarity of a person's attitude to certain values and his / her readiness to focus on them in situations of internal choice. The specific features of personal experiences and his / her behaviour in light of personal understanding of various situations [7; 11] defines his / her emotional and values-based attitude. This is the most difficult component to assess, which is considered as an adopted (through his / her relationships with other people, from various information sources) attitude to nature, other people (society), labour, profession, homeland (and world as a whole).

Considerable teaching experience of the authors shows that the sentence completion method can be a high-quality tool for assessing the formation of the environment-oriented component of competencies, which envisages gaining experience of emotional and valuesbased attitude within the scope of students personal values. This method can provide information about the activity-based (behavioural) component of emotional and valuesbased attitude, as well as indirect outcomes of teaching [13; 15].

The strengths of this method are its flexibility and adaptability to various research tasks. Group observation is possible. According to experts, the validity and reliability of this type of projective methods is quite high (if the number of respondents is large enough). The questioning is voluntary, respondents are warned that they may not answer the questions.

Questionnaire.

Dear participant, please complete the following sentences:

1. When I think about nature, I imagine ...

2. The major environmental problem is ...

3. If I could solve only one environmental problem, I would choose ...

4. To save electricity, my family can ...

5. The primary reason people do not to participate in environmental activities is ...

The responses received were analysed according to the following aspects:

- changes in the nature and intensity of emotional reaction characterising the sensitivity to the sensuous and expressive elements of natural objects (emotional aspect);

- changes in the cognitive manifestations that determine the motivation and focus of cognitive activity in relation to natural objects (cognitive / ontological aspect);

- changes in the nature of practical interaction with natural objects, from receiving benefits from them to accepting nature as a value (practical / activity-based aspect);

- totality of directed actions, i.e. external (motor) and internal (mental) activity when interacting with natural objects, solving environmental problems and addressing conflict situations from self-interest to professionally focused activity (behavioural / creative aspect). 
The expressiveness of the response, nature and intensity of emotional and cognitive comments, reflection of practical (pragmatic) inclinations, activity-based (behavioural) interactions with natural objects were taken in the completed sentences as the indicators of the developed emotional and values-based attitude. Analysing the results of the study carried out using the projective method of sentence completion, the authors identified three levels of emotional and values-based attitude to nature: low, medium and high.

The features of the low level of emotional and values-based attitude to nature (for all sentences) are:

- refusing to answer or crossing out the sentences, which show disinterest of respondents, their unwillingness to understand the task and to fulfil it carefully, other reasons -0 points;

- giving run-around responses, which indicate insufficient knowledge of the conceptual framework (terminological confusion) and low awareness of the basics of ecology. In these responses, there are no cognitive manifestations that determine the motivation and focus of cognitive activity in relation to natural objects (cognitive aspect), and the emotional reaction characterising the sensitivity to the sensuous and expressive elements of natural objects is represented by emotionally unconscious signal. The main emphasis in the responses is placed on the practical / pragmatic attitude to nature as a "useful product". At that the respondents demonstrate a lack of understanding the scope of the problem (nature is seen only as an object for one's own benefit and considered as nothing more than a means of satisfying desires and needs of a person) and refuse to address and solve environmental problems (anthropocentrism). Within this level, one completed sentence is estimated at 1 point.

The medium level of emotional and values-based attitude to nature is visible in the responses with a traced cognitive aspect. Notably, the authors assumed that the respondent may make some mistakes in environmental concepts. The responses have a pronounced emotional reaction, and the perception of a natural object as a "useful product" for maintaining life and comfort is emotionally loaded. Additionally, the responses related to this level has an aftereffect or follow-up, a kind of contextuality (the respondents may emotionally react to the events covered in various mass media and strongly impressed them on the eve of receiving the questionnaire). However, there is some inconsistency at this level of emotional and values-based attitude to nature, so a completed sentence scores 2 points.

Consistent attitude to nature (i.e. nature is inherently valuable) implies the high level of emotional and values-based attitude to nature. Two viewed aspects of emotional and values-based attitude (cognitive and actional components, cognitive and affective, etc.) are jointly found in the responses, which can be regarded as a nature-centred core of the personal experience. The respondents perfectly understand the roots of the problem, soundly reason them, give consistent responses and justify ideas. These are the responses that highlight and reveal the qualified insights of the respondents (focus of cognitive activity). Moreover, the responses with noted importance (impact) of environmental education are assigned to this level. Therefore, one completed sentence at the high level is estimated at 3 points.

When analysing the questionnaires, the authors factored in the points scored for all sentences. Summing up the received points, they got the following results:

- $\quad$ low level of emotional and values-based attitude (0-6 points);

- medium level of emotional and values-based attitude (7-11 points);

- $\quad$ high level of emotional and values-based attitude (12-15 points).

The article presents the summarised and examined findings of experimental work carried out in 2016 - 2021. The questionnaire was completed by students of Penza State 
University (V.G. Belinsky Institute of Teacher Education and Medical Institute) and students of A.V. Khrulev Penza Branch of the Military Academy of Logistics.

The responses to the sentence "When I think about nature, I imagine ..." are analysed on the basis of comparison, and then expansion of indicators illustrating the intensity of emotional reaction, peculiarity of cognitive manifestations, nature of practical interaction and activity in nature.

The low level of emotional and values-based attitude is distinguished by refusing to answer or crossing out the sentences, as well as providing inconsistent diagnostic indicators. The nature and intensity of emotional reaction is manifested through emotionally unconscious signals, with the elements of responses indicating insufficient knowledge of the basics of ecology. For example: "... everything that inhabits our vast nature"; "... a planet with a large amount of natural world"; "... my future"; “... how beautiful Russian nature is!”, “... beautiful forests, clean air, everything blooming!”; “... myself as an unusually outgoing, light-hearted, free and independent person"; "... the world, where everything lives and flourishes". The nature of the practical interaction is assessed through the pragmatism in the response, e.g. expecting and deriving benefit from a natural object. Natural objects are described as a "useful product": “... a field sown with corn", “... wheat fields" (anthropogenic ecosystems). The behaviour in nature at this level of emotional and values-based attitude can be assessed as selfish. The focus of activity in nature characterises the consumer perception of nature to support life and personal comfort (anthropocentrism), for example, “... garden party”, “... campfire”, “... picking berries and mushrooms", “... vacation at sea”, "... fishing”. There are no responses that convey cognitive activity of the respondents in relation to natural objects.

The responses with coloured images testify to the medium level of emotional and values-based attitude to nature. They are coherent according to the major diagnostic indicators: emotional perception accompanies the description of natural objects and their relationship with the environment: “... a blooming meadow", “... singing birds", “... a blue river". The responses contain the names of animate and inanimate natural objects in their intrinsic value, i.e. denominative elements: “... mountains”, “... rivers”, “... seas”, “... squirrels", “... bears”, “... birches”, “... oaks”, as well as give a holistic view of nature and interconnection of natural objects: “... ducks with ducklings, graylings going up the mountain rivers"; “... mammals with cubs". Such responses are regarded as a manifestation of cognitive activity. The nature of practical interaction determines the emotional perception of a natural object as a "useful product" (or an anthropogenic ecosystem instead of a natural ecosystem). For example, " ... a blooming apple orchard with singing birds", “... a forest with sky-high trees, reservoirs with clear water, fields sown with wheat", "... a forest in the mind of a perfectionist", “... a village pond located in a ravine, a strip of birches in 3-4 rows at the top of a ravine, and endless fields behind them", “... the weather is clear, not a cloud in the sky, the field smells of mint and strawberries, the river is not far away with a forest beyond it, a village can be seen on the hill, a light wind bears a smell of smoke from the chimney of the bathhouse". Activities in nature presuppose a professional focus, but some elements of the responses indicate a lack of understanding of the environmental effects: “... possible use of nuclear, chemical and biological weapons".

The high level of emotional and values-based attitude is illustrated by a consistent (at least two elements, for example, cognitive and emotional, practical and emotional, etc.) perception of natural objects and professional focus of ideas. There are rational responses (voicing reason, intelligence, logic, rationality), comprehensive images of nature with an emotional signal. For example: "... coniferous forest with mountains against the background of sunrise or sunset, fields, lakes, rivers, forest paths", "forests, singing birds, rustling leaves", “... a spring forest, namely its edge, with large powerful trees, a meadow disappearing over the horizon, a shimmering river in the golden sunset, serenity"; “... that 
nature is like a medicine for me, sometimes it calms, for example, when you go up a mountain or swim in a river, this is so good for health"; “... everything around me, except for buildings".

The completion of the sentence "The major environmental problem is..." is interpreted by changes in the type of cognitive manifestations.

The features of the low level of emotional and values-based attitude to nature are the refusal to answer and lack of knowledge about environmental problems or their denial ("... the tropics"; "... there are none", “... the greed of a human”). This level also include the responses with weak arguments and conventional ideas, which indicate insufficient knowledge of the basics of ecology, for example, "... ecological pollution by industrial enterprises", “... everyone should know that pollution of nature harms human health", “... warming".

Within the medium level of emotional and values-based attitude to nature, the responses reflect:

- examples of local, regional, and global environmental problems or their overview, for example, "pollution of the Sura River”, “... melting glaciers”, “... garbage”, “... air and soil pollution", “... ozone holes", “... pollution of forests, fields, reservoirs with household waste", “... high deforestation and heavy industrial air pollution”;

- contextuality (emotional response to information from various mass media, political propaganda, etc.): “... ozone depletion on the earth", “... air pollution”, “... melting glaciers", “... river pollution, as all factories dump their waste into rivers";

- emotionally loaded assessments of the existing environmental problems with marked role of a human (“... people and their improper use of tools", “... pollution of the environment by humans themselves", “... air pollution due to factories, exhaust emissions, unscrupulous people who throw rubbish everywhere, burn trees, which generate oxygen, pollute water reservoirs").

The high level of emotional and values-based attitude to nature is defined by knowledge of environmental problems tackled by non-governmental organisations and problems of environmental education, for example, “... drying up of the Aral Sea", “... survival of the Amur tiger", “... a society that is indifferent to environmental problems". At the same time, the environmental problems identified in the responses are professionally focused (".. military conflicts around the world", “... environmental pollution by nuclear and chemical waste", “... possible use of nuclear, chemical and biological weapons).

When completing the sentence "If I could solve only one environmental problem, I would choose ...", many questionnaire participants give responses related to the previous task. Therefore, the characteristics of the levels of emotional and values-based attitude to nature are similar according to the type of cognitive manifestations. However, the responses suggest different approaches to handling the environmental problems, which determines the behaviour of the respondents.

Values-based attitude to nature is seen at the low level, if the respondents:

- refuse to answer or miss the sentence;

- give implausible responses testifying to insufficient knowledge of the essence of the described event, object, phenomenon (“... solving all global problems of ocean pollution”, “... destruction of nuclear weapons", etc.);

- give stock responses that indicate insufficient knowledge of the basics of ecology, for example “... purity of nature", “... problem of pollution”, “... cleaning of the environment";

- give run-around responses, which evidence passive interest in solving environmental problems and weak citizenship (“... I am not responsible for this", “... the state should combat such problems", “... the most important", “... water scarcity in African countries"). 
Emotional and values-based attitude is considered at the medium level if the respondents examine the current situation in the global, regional and local terms and make proposals for viable ways to tackle the environmental problem. For example: “... initiating household waste management in Russia and reducing nature and air pollution to a greater extent", “... air pollution due to exhaust emissions", “... cleaning the city streets from waste", “... waste disposal".

At the high level of emotional and values-based attitude to nature, the respondents display a professional focus in solving environmental problems, identify their causes and highlight environmental education challenges to be addressed. Here are some of the responses: "... destruction of synthetic waste that do not biodegrade in the soil", "... the essential one, the solution of which may greatly influence the life of people, or banning chemical plants", “... work on minimising hazards and recycling waste of hazardous industries, inkling the military one", “. . analysis of the negative environmental impact of enterprises producing hazardous products", “... creating a group of people that will be engaged in cleaning the environment and entrap perpetrators", "... impact of hostilities on ecosystems", “... not causing such problems on our own, changing attitude of other people to environmental problems".

The results of completing the sentence "To save electricity, my family can ..." are used to mark the ways of handling environmental problems and determine the behavioural reactions of the respondents. The authors also identified three levels of emotional and values-based attitude to nature.

The low level is noted among the respondents, who refuse to discuss and analyse the problem, tell jokes or misunderstand the essence of the problem: “... not abandon it", “... not fall out of using electricity, because most of essential equipment is powered", "... use a torch", “... fall out of watching TV", “... live without turning on the light", “... use computers less, but I think that it will not work out in our time".

Withing the medium level of emotional and values-based attitude to nature, the respondents suggest minimising demands in terms of comfort, but do not specify actions, for example, “... rationally use the supplied electricity”, “... use lighting less often”, “... use the Internet and electrical appliances less often".

The features of the responses at the high level include suggestion conscious actions, voicing professional focus in saving (conserving) resources, as well as submitting proposals for the use of alternative energy sources. For example: “... use solar panels", “... install solar panels", “... independently make a wind turbine to generate electricity”, “... avoid using unnecessary energy consumers", “... unplug everything when leaving the house and turn off the light when you do not need it", “... use energy-saving bulbs, participate in Earth Hour movement". One of the questionnaire participants replied in verse form:

"It is simple, saving energy,

We need little: living cleverly."

The last completed sentence "The primary reason people do not to participate in environmental activities is ..." is examined as follows.

The low level of emotional and values-based attitude to nature is revealed through:

- refusing to answer or omitting the sentence;

- misunderstanding the essence of the topic covered in the questionnaire ("... environmental resource", “... air pollution", “... littering”, “... use of chemicals”, “... wipers should do their work");

- giving run-around responses: “... busy life”, “... lack of free time”;

- demonstrating insufficient knowledge of concepts and basics of ecology ("... that people do not place emphasis on ecology", “... absence of powerful protection laws".

At the medium level of emotional and values-based attitude, the respondents: 
- clearly understanding the problem, not correctly committing to writing, so giving an inappropriate response. For example: “... laziness, living for a day and disregarding future generations", "... ignorance of ecology and poor awareness of what is happening around", " $\ldots$ that people ignore life, they are not interested in nature", “... that people think that someone else will solve these problems", "... thought that they will not change anything anyway", "... making a profit on ecology, striking gold at any cost".

- $\quad$ giving terse responses, just acknowledging the fact without examining its reasons (“... lack of discipline", “... lawlessness", “... laziness, indifference”, “... progress, material benefits, greed of people", “... that they litter", “... indifference”, “... lack of personal interest").

If the questionnaire participants see the essence of the problem, reasonably justify their point of view, use professional concepts and note the impact of environmental education, then their emotional and values-based attitude to nature is at the high level. For example: "... not understanding the further effects of environmental problems", “... their misperception that they do not affect the environment anyway", “... lack of interest and need for this type of activity, it is easier to turn a blind eye than to restore something", "... laziness and ignorance of what may happen, if you refrain from participating in environmental activities", “... laziness, interest in other issues (a different value paradigm)", “... personal irresponsibility, the fact that not everyone understands that he / she can make a difference", "... laziness and lack of understanding that they do not want to help themselves".

The results of the study using the projective method of sentence completion indicate an expected transition from a crude pragmatic perception of natural objects and environmental problems to an emotionally loaded and holistic perception of nature in its inherent value. Consequently, the professional vision of ways to solve environmental problems and to address challenges in environmental education is developed. Such pilot study enables:

- determining the existing level of formed experience of emotional and values-based attitude to nature in a specific group of respondents;

- correlating the identified level of emotional and values-based attitude to nature with emotional and values-based attitude (public and personal) to homeland.

\section{Conclusion}

The research shows that purposeful educational activities aimed forming the environmentoriented component of competencies result in the transformation in students from a crude utilitarian perception of natural objects and environmental problems to a holistic perception of nature, recognition of its intrinsic value and professional vision of solutions to environmental problems. The data obtained confirm the views of most researchers about the relationships in the chain of values-beliefs-intentions and ecologically significant behaviours of a person. Only the analysis of the aggregate data obtained and their review in the system and integrity enable drawing final conclusions about the effective formation of the environment-oriented component of competencies.

\section{References}

1. O.N. Vasina, Bulletin of Higher Education Institutions, 4 (2009)

2. O.N. Vasina, O.N. Ponomariova, S.V. Shurygin, Modern Challenges in Science and Education, 4 (2018)

3. O.N. Vasina, O.N. Ponomariova, D.A. Shpynev, Ecology. Drill Book (Penza, 2017) 
4. S.D. Deryabo, V.A. Yasvin, Methods of diagnosing and correcting attitude to nature (Moscow, 1995)

5. E.N. Dzyatkovskaya, Modern Teacher Training, 6 (2020)

6. A.N. Zakhlebny, E.N. Dzyatkovskaya, I.V. Wagner, A.Yu. Liberov, Environmental Education: before School, at School, after School, 2 (2012)

7. E.A. Konova, Bulletin of the South Ural State University, 5, 3 (2013)

8. Authoring team, Best practices of environmental education for sustainable development (St. Petersburg, 2017)

9. V.V. Nikolina, Human and Education, 1 (2020)

10. V.I. Panov, Psychological Diagnosis, 1 (2012)

11. M.A. Petukhova, O.M. Krivoshapkina, Modern Challenges in Science and Education, 6 (2019)

12. O.N. Ponomariova, O.N. Vasina, D.E. Salov, Youth. Education. Science, 1 (2020)

13. O.N. Vasina, O.N. Ponomariova, S.V. Shurygin, Ecopsychological Research: Ecology of Childhood and Psychology of Sustainable Development, 6 (2020)

14. O.N. Ponomariova, O.N. Vasina, E.V. Kolesova, Ecology. Teacher's Book (Penza, 2017)

15. O.N. Ponomariova, A.A. Tselykovskikh, O.N. Vasina, I.I. Grachev, Bulletin of Higher Education Institutions, 3 (2016)

16. O.N. Ponomariova, Methodical system of teaching ecology in secondary school: Thesis (Penza, 2000)

17. V.A. Romanov, Alphabetical list of topics for doctoral theses in pedagogy and psychology in 1937-2017 (Tula, 2018)

18. O.V. Selezneva, N.A. Mamaeva, Ecopsychological Research: Ecology of Childhood and Psychology of Sustainable Development, 6 (2020)

19. O.N. Vasina, O.N. Ponomariova, European Proceedings of Social and Behavioural Sciences, 90 (2018)

20. O.N. Ponomariova, O.N. Vasina, International Journal of Environmental and Science Education, 11, 15 (2016) 